\title{
ON THE WEAKNESS OF SOME BOUNDARY COMPONENT
}

\author{
TOHRU AKAZA
}

1. Let $D$ be a domain in the complex $z$-plane and $r$ be a boundary component of $D$ consisting of a single point. The component $r$ is said to be weak if its image under any conformal mapping of $D$ consists of a single point. If $\gamma$ is not weak, then we say that $\gamma$ is unstable (Sario [3], [4]).

Let $S_{n}(n=1,2, \ldots)$ be a sequence of slits being symmetric and orthogonal to the positive real axis of the complex $z$-plane and converging to the origin $O: z=0$. We delete the set $\bigcup_{n=1}^{\infty} S_{n} \cup\{O\}$ from the $z$-plane and denote by $D$ the resulting domain. In this note, we treat the weakness of the boundary component $O$ of the domain $D$.

2. First we prove the following

Lemma 1. Consider two slits: $x=a_{j}(>0),|y| \leqq h_{j}(j=1,2),\left(a_{2}<a_{1}\right)$ which are symmetric and orthogonal to the positive real axis and satisfy the equality $\frac{h_{1}}{a_{1}}=\frac{h_{2}}{a_{2}}=k$. Construct a doubly connected domain $B$ bounded by two circular arcs $C_{j}:|z|=\sqrt{a_{j}^{2}+h_{j}^{2}} \alpha \leqq, \arg z \leqq 2 \pi-\alpha,(j=1,2)$, where $0<\alpha=\tan ^{-1} k\left(<\frac{\pi}{2}\right)$, and slits $S_{1}, S_{2}$. Let $\mu$ be the module of $B$ and $\mu^{*}$ be the module of the ring domain $R: \sqrt{a_{2}^{2}+h_{2}^{2}}<|z|<\sqrt{a_{1}^{2}+h_{1}^{2}}$. Then it holds

$$
\frac{1}{M(\alpha)} \mu^{*} \leqq \mu \leqq M(\alpha) \mu^{*},
$$

where $M(\alpha)$ is a constant depending only on $\alpha$.

Proof. Let $z_{j}=a_{j}+i h_{j}$ and $z_{j}^{\prime}=a_{j}-i h_{j}$ be two endpoints $S_{j}(j=1,2)$. We map the trapezoid $T:\left(z_{2}, z_{2}^{\prime}, z_{1}^{\prime}, z_{1}\right)$ onto the quadrilateral $\left(z_{2}, z_{2}^{\prime}, z_{1}^{\prime}, z_{1}\right)$ bounded by two minor circular arcs ${\widetilde{z_{2}}}_{z_{2}^{\prime}}^{\prime}$ on $|z|=\sqrt{a_{2}^{2}+h_{2}^{2}},{\overparen{z_{1}}}_{z_{1}^{\prime}}^{\prime}$ on $|z|=\sqrt{a_{1}^{2}+h_{1}^{2}}$ and two rectilinear segments $\overline{z_{2} z_{1},}, z_{2}^{\prime} z_{1}^{\prime}$ under the topological mapping $\zeta(z)=\sqrt{\left(1+k^{2}\right) x^{2}-y^{2}}$ $+i y=\sqrt{x^{2} \sec ^{2} \alpha-y^{2}}+i y, z=x+i y$. It is obvious that $|\zeta(z)|=\sqrt{1+k^{2}} x$.

Received June 29, 1960. 
Now we put

$$
p=\frac{1}{2}\left(\frac{\partial \zeta}{\partial x}-i \frac{\partial \zeta}{\partial y}\right), \quad q=\frac{1}{2}\left(\frac{\partial \zeta}{\partial x}+i \frac{\partial \zeta}{\partial y}\right) .
$$

By an easy computation, we have

$$
\begin{aligned}
\frac{|p|+|q|}{|p|-|q|} & \leqq \frac{\left(x \sec ^{2} \alpha+\sqrt{x^{2}} \sec ^{2} \alpha-y^{2}\right)^{2}+y^{2}}{x \sec ^{2} \alpha \sqrt{x^{2} \sec ^{2} \alpha-y^{2}}} \leqq \frac{\left(\sec ^{2} \alpha+\sec \alpha\right)^{2}+\tan ^{2} \alpha}{\sec ^{2} \alpha} \\
& \leqq \frac{4 \sec ^{4} \alpha+\sec ^{2} \alpha}{\sec ^{2} \alpha}=4 \sec ^{2} \alpha+1 .
\end{aligned}
$$

If we put $4 \sec ^{2} \alpha+1=M(\alpha)$, then

$$
\sup _{z \in T^{\prime}} \frac{|p|+|q|}{|p|-|q|} \leqq M(\alpha)
$$

Hence $\zeta(z)$ is a quasiconformal mapping with bounded dilatation. Therefore, if we define

$$
\zeta=\varphi(z)=\left\{\begin{aligned}
\zeta(z), & z \in T \\
z, & z \in B-T,
\end{aligned}\right.
$$

then $\zeta=\varphi(z)$ is a quasiconformal mapping of $B$ onto $R$ with bounded dilatation. Thus we have the required inequality

$$
\frac{1}{M(\alpha)} \mu^{*} \leqq \mu \leqq M(\alpha) \mu^{*}
$$

3. Suppose that $S_{n}(n=1,2, \ldots)$ are segments : $x=a_{n}(>0),|y| \leqq h_{n}$ satisfying $0<a_{n+1}<a_{n}, \lim _{n \rightarrow \infty} a_{n}=0$ and

$$
h_{n} \leqq a_{n} \tan \alpha=h_{n}^{\prime}
$$

for some fixed $\alpha\left(0<\alpha<\frac{\pi}{2}\right)$.

Let $S_{n}^{\prime}$ be a segments $x=a_{n},|y|=h_{n}^{\prime}$. Denote by $D$ (or $D^{\prime}$ ) the domain obtained by deleting segments $S_{n}$ (or $\left.S_{n}^{\prime}\right) \quad(n=1,2, \ldots)$ and the origin $z=0$ from the complex $z$-plane. It is obvious that $D \supset D^{\prime}$.

We construct doubly connected domains $B_{n}(n=1,2, \ldots)$ in $D^{\prime}$ bounded by $S_{n}^{\prime}, S_{n+1}^{\prime}$ and by two circular $\operatorname{arcs} C_{j}:|z|=\sqrt{a_{j}^{2}+h_{j}^{\prime 2}}, \alpha \leqq \arg z \leqq 2 \pi-\alpha$, $(j=n, n+1)$. Evidently, $B_{n} \subset D$ and $B_{n} \cap B_{m}=\phi$ if $n \neq m$. Let $\mu_{n}$ be the module of $B_{n}$. By Lemma 1, we have 


$$
\frac{1}{M(\alpha)} \mu_{n}^{*} \leqq \mu_{n} \leqq M(\alpha) \mu_{n}^{*}
$$

where $\mu_{n}^{*}=\log \frac{a_{n}}{a_{n+1}}$ is the module of the ring domain $\sqrt{a_{n+1}^{2}+h_{n+1}^{\prime 2}}<|z|<$ $\sqrt{\boldsymbol{a}_{n}^{2}+h_{n}^{\prime 2}}$. Hence it follows that

$$
\sum_{n=1}^{\infty} \mu_{n}^{*} \leqq M(\alpha) \sum_{n=1}^{\infty} \mu_{n}
$$

Since $\lim _{n \rightarrow \infty} a_{n}=0$, the left hand side of the above inequality is divergent. By Savage's criterion [5] we see that the origin $O$ is a weak boundary component of $D$. Thus we obtain the following

Theorem 1. If $S_{n}(n=1,2, \ldots)$ are segments: $x=a_{n}(>0),|y| \leqq h_{n}$ satis. fying $0<a_{n+1}<a_{n}, \lim _{n \rightarrow \infty} a_{n}=0$ and

$$
h_{n} \leqq a_{n} \tan \alpha=h_{n}^{\prime}
$$

for some fixed $\alpha\left(0<\alpha<\frac{\pi}{2}\right)$, then $O$ is a weak boundary component of the domain obtained by deleting $\bigcup_{n=1}^{\infty} S_{n} \cup\{O\}$ from the z-plane.

4. Here we show that in the case when segments in our Theorem 1 do not satisfy the condition $(*)$ the origin $O$ is not always weak.

First we prove the following

LEMma 2. Consider two slits $S_{j}: x=a_{j}(>0),|y| \leqq h_{j}(j=1,2),\left(a_{2}<a_{1}\right)$ which are symmetric and orthogonal to the real axis. Let $\Omega$ be the doubly connected domain obtained by deleting $S_{1}$ and $S_{2}$ from the z-plane and let $Q$ be the rectangle: $\left(a_{2}+i h, a_{2}-i h, a_{1}-i h, a_{1}+i h\right)$, where $h=\operatorname{Min}\left(h_{1}, h_{2}\right)$. If 4 is the module of $\Omega$, then it holds

$$
\mu \leqq \frac{\pi\left(a_{1}-a_{2}\right)}{h}
$$

Proof. We denote by $\{r\}$ a family of rectifiable curves in $\Omega$ separating $S_{1}$ from $S_{2}$ and by $\left\{r^{\prime}\right\}$ a family whose elements consist of rectifiable curves

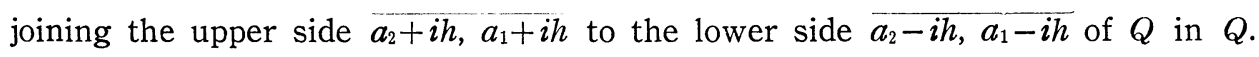
It is obvious that each $r \in\{r\}$ contains a curve $r^{\prime} \in\left\{\gamma^{\prime}\right\}$. Denoting by $\lambda\{r\}$, $\lambda\left\{\gamma^{\prime}\right\}$ the extremal lengths of these families in the sense of Ahlfors-Beurling [1], we get the following inequality: 


$$
\lambda\left\{\gamma^{\prime}\right\} \leqq \lambda\{r\}
$$

From the relation $\lambda\{r\}=\frac{2 \pi}{\mu}$ and $\lambda\left\{r^{\prime}\right\}=\frac{2 h}{a_{1}-a_{2}}$, we have

$$
\mu \leqq \frac{\pi\left(a_{1}-a_{2}\right)}{h}
$$

Now we denote by $S_{n}(n=1,2, \ldots)$ segments in the $z$-plane

$$
x=\frac{1}{n},|y| \leqq h_{n}=c\left(\frac{1}{n-1}\right)^{p}, \quad(0<p<1)
$$

where $c$ is a positive constant and $z=x+i y$. Let $D$ be a domain obtained by deleting $\bigcup_{n=1}^{\infty} S_{n} \cup\{O\}$ from the $z$-plane and let $B_{j}(j=1,2, \ldots)$ be any sequence of doubly connected domains in $D$ separating $O$ from the infinity and converging to $O$. We suppose that $B_{j+1}$ lies in a domain $G_{i}$ which is a component, containing $O$, of the complementary sets of $\overline{B_{j}}$ with respect to the $z$-plane.

Let $S_{m(j)}$ be the segment such that, for any $n>m(j), S_{n} \subset G_{j}$ and that $S_{m(j)} \neq G_{j}$. Then $B_{j}$ separates $S_{n}(n>m(j))$ from $S_{m(j)}$.

Without loss of generality, we may assume that $\left\{B_{j}\right\}\left(j=k_{l}+1, \ldots, k_{l+1}\right)$ are all the doubly connected domains separating $S_{m\left(k_{l}+1\right)}\left(=\cdots=S_{m\left(k_{l+1}\right)}\right)$ from $S_{m\left(k_{l}+1\right)+1}$, where $k_{0}=0, \boldsymbol{m}(1)=1$ and

$$
m\left(k_{l+1}\right)<m\left(k_{l}+1\right)+1 \leqq m\left(k_{l+1}+1\right) .
$$

Denote by $\Omega_{l}$ the domain obtained by deleting $S_{m\left(k_{l}+1\right)}$ and $S_{m\left(k_{l}+1\right)+1}$ from the $z$-plane. Then $B_{j}\left(j=k_{l}+1, \ldots, k_{l+1}\right)$ are contained in $\Omega_{l}$. The well-known Teichmüller's inequality implies that

$$
\sum_{j=l_{l}+1}^{k_{l+1}} \mu_{j} \leqq \mu_{l}^{*}
$$

where $\mu_{j}\left(j=k_{l}+1, \ldots, k_{l+1}\right)$ are the moduli of $B_{j}\left(j=k_{l}+1, \ldots, k_{l+1}\right)$ and $\mu_{l}^{*}$ is that of $\Omega_{l}$.

Thus, using Lemma 2, we obtain

$$
\begin{aligned}
& \sum_{j=1}^{\infty} \mu_{j}=\sum_{l=0}^{\infty} \sum_{j=k_{l}+1}^{k_{l+1}} \mu_{j} \leqq \sum_{l=0}^{\infty} \mu_{l}^{*} \\
& \leqq \pi \sum_{l=0}^{\infty} \frac{\frac{1}{m\left(k_{l}+1\right)}-\frac{1}{m\left(k_{l}+1\right)+1}}{h_{m\left(k_{l}+1\right)+1}} \leqq \pi \sum_{n=1}^{\infty} \frac{\frac{1}{n}-\frac{1}{n+1}}{h_{n+1}} \leqq \frac{\pi}{c} \sum_{n=1}^{\infty} n^{p-2} .
\end{aligned}
$$


Since $\sum_{n=1}^{\infty} n^{p-2}(0<p<1)$ is convergent, we see that the series $\sum_{j=1}^{\infty} \mu_{j}$ is convergent for any sequence $\left\{B_{j}\right\}$. By using Oikawa's theorem [2], i.e., the converse of Savage's criterion, we have the following

Theorem 2. If $S_{n}(n=1,2, \ldots)$ are segments: $x=\frac{1}{n},|y| \leqq c\left(\frac{1}{n-1}\right)^{p}$, $(0<p<1)$, then the origin $O$ is an unstable boundary component of the domain obtained by deleting $\bigcup_{n=1}^{\infty} S_{n} \cup\{O\}$ from the z-plane.

Recently Oikawa has treated the case that the number of boundary components converging to the origin is not countable and obtained interesting results, some of which contain our results.

\section{REFERENCES}

[1] Ahlfors, L. V. and Beurling, A., Conformal invariants and function-theoretic nullsets, Acta Math., 83 (1950), 101-129.

[2] Oikawa, K., On the stability of boundary component, Pacific Jour. Math., 10 (1960), 263-294.

[ 3 ] Sario, L., Stability problems on boundary components, Proc. Conference Analytic Function, Princeton (1957), 55-72.

[4] Sario, L., Strong and weak boundary components, Jour. Analyse Math., 5 (1958), 389398.

[5] Savage, N., Weak boundary components of an open Riemann surface, Duke Math. Jour., 24 (1957), 79-95.

\section{Mathematical Institute}

Kanazawa University 